\title{
Properties of Carbon-Oxygen White Dwarf Merger Remnants
}

\author{
Chenchong Zhu ${ }^{1}$, Philip Chang ${ }^{2}$, Marten van Kerkwijk ${ }^{1}$, and \\ James Wadsley ${ }^{3}$ \\ ${ }^{1}$ Department of Astronomy \& Astrophysics, University of Toronto, \\ 50 St. George Street, Toronto, Ontario, Canada, M5S 3H4 \\ ${ }^{2}$ Department of Physics, University of Wisconsin-Milwaukee, \\ 1900 E Kenwood Blvd., Milwaukee, Wisconsin 53211, USA \\ ${ }^{3}$ Department of Physics \& Astronomy, ABB-241, McMaster University, \\ 1280 Main St. W, Hamilton, Ontario, Canada, L8S 4M1 \\ Correspondence email: cczhu@astro.utoronto.ca
}

\begin{abstract}
Recent studies have shown that for suitable initial conditions both super- and subChandrasekhar mass carbon-oxygen white dwarf mergers produce explosions similar to observed $\mathrm{SNe}$ Ia. The question remains, however, how much fine tuning is necessary to produce these conditions. We performed a large set of SPH merger simulations, sweeping the possible parameter space. We find trends for merger remnant properties, and discuss how our results affect the viability of our recently proposed sub-Chandrasekhar merger channel for SNe Ia.
\end{abstract}

Keywords. stars: white dwarfs, supernovae — binaries: close - methods: n-body simulations

\section{Introduction}

The nature of Type Ia supernovae (SNe Ia) progenitors, and their evolution leading up to the explosion, are still not yet well understood (Van Kerkwijk et al. 2010). The most widely accepted scenario is that of a carbon-oxygen white dwarf (CO WD) in a binary that acquires mass through either accretion from a non-degenerate companion (the single-degenerate channel) or merger with another WD (the double-degenerate channel), until it approaches the Chandrasekhar mass, $\mathrm{M}_{\mathrm{Ch}}$ (Howell 2011). This scenario still has several unresolved issues, including discrepancies between the rates of SNe Ia and progenitors (Maoz 2008; Ruiter et al. 2009), the lack of sufficient numbers of supersoft sources for the single-degenerate (and potentially double-degenerate) channel (Di Stefano 2010a,b; Gilfanov \& Bogdán 2010), and the still-poorly understood nature of the deflagration-to-detonation transition, required for $\mathrm{M}_{\mathrm{Ch}}$ explosions to look like observed SNe Ia (Howell 2011). These issues suggest the utility of considering alternate SNe Ia channels, for instance the proposed channels of Pakmor et al. (2010), Guillochon et al. (2010), and, for this paper, Van Kerkwijk et al. (2010).

Van Kerkwijk et al. propose an SN Ia channel that involves the merger of two CO WDs whose total mass is below $\mathrm{M}_{\mathrm{Ch}}$. As shown in Lorén-Aguilar et al. (2009), the merger between two equal mass $0.6 \mathrm{M}_{\odot}$ WDs results in a massive, uniformly rotating core with a differentially rotating, sub-Keplerian, fat disk. While the merger is hottest at the center of the core, it is not hot enough to ignite runaway carbon fusion. The disk then accretes onto the core on a characteristic timescale of several hours. The accretion compresses the core, driving up temperatures until the accretion and carbon fusion timescales are comparable. The neutrino cooling timescale is six orders of magnitude longer, and therefore a carbon fusion runaway results. 
This channel possesses a number of advantages. If most merging CO WD binaries (regardless of total mass or mass ratio) are assumed to result in SNe Ia, it would account for the observed SNe Ia rate (Van Kerkwijk et al. 2010). Since lower-mass CO WDs generally come from lower-mass stars, it also explains the observed decrease of typical SN Ia luminosity with increasing host stellar population age. Sim et al. (2010) recently showed that sub-Chandrasekhar detonations can produce light curves and spectra very similar to observed SNe Ia, without resorting to a deflagration-to-detonation transition. This channel stands in contrast to He-CO sub- $\mathrm{M}_{\mathrm{Ch}}$ merger channels, which involve the detonation of a helium layer around a sub- $\mathrm{M}_{\mathrm{Ch}} \mathrm{CO} \mathrm{WD}$, producing light curves and spectra that do not resemble observed SNe Ia (Sim et al. 2010; Howell 2011; see e.g. Woosley \& Kasen 2011 on whether or not the effect of helium can be minimized).

In order to determine the viability of Van Kerkwijk et al.'s analytical sketch, it must be determined which regions of the CO WD merger parameter space will lead to detonations, and whether, e.g., nearly equal mass WDs are required, and, if so, how close to unity the mass ratio must be.

We have run a series of 45 high-resolution smoothed-particle hydrodynamic (SPH) simulations of white dwarf mergers, exploring a broad range of parameter space to seek global trends in merger remnant properties as a means of interpolating between numerical results. Our results can be used to create initial conditions for models of the further viscous and thermal evolution of the remnants (e.g. Shen et al. 2011) to determine if any of them reach conditions necessary for carbon detonation. They also have utility outside our own project, as they would allow anyone to estimate the properties of a CO WD merger remnant without having to simulate the merger themselves.

This report focuses on the implications of our results for the sub- $\mathrm{M}_{\mathrm{Ch}}$ merger channel. Details regarding trends and a possible semi-analytical picture of CO WD mergers will be presented in a forthcoming publication.

\section{Code and Initial Conditions}

We used the SPH code Gasoline (Wadsley et al. 2004). Gasoline uses the formulation for artificial viscosity in Monaghan (1992), augmented with a Balsara (1995) switch and a method that reduces the artificial viscosity coefficient $\alpha$ when no shocks are present. We have modified Gasoline to use the Helmholtz equation of state (Timmes 2010, details in Timmes \& Swesty 2000). No nuclear reaction network was used, as previous simulations have shown that nuclear processing is not important to the hydrodynamic evolution of lower-mass mergers (Guerrero et al. 2004; Lorén-Aguilar et al. 2009). An in situ detonation, however, may arise during mergers between nearly equal-mass CO WDs where the accretor has mass $\gtrsim 0.9 \mathrm{M}_{\odot}$ (Pakmor et al. 2010, 2011).

Our input binaries are composed of $8 \times 10^{4}$ to $4 \times 10^{5}$ particles, depending on their mass. White dwarfs were relaxed individually and given equilibrium temperatures of $\sim 10^{7} \mathrm{~K}$. Their initial separation is chosen such that the lower-mass binary fills its Roche lobe. Since the synchronization timescale is expected to be much longer than the angular momentum loss timescale due to gravitational radiation, our binary is unsynchronized at the start of merger (Marsh et al. 2004, but see their discussion and Fuller \& Lai 2011).

\section{Results}

The merger remnants for all our runs consist of a core and a sub-Keplerian disk. For highly unequal mass mergers, almost the entire accretor forms 85 - $90 \%$ of the core, while $\sim 70 \%$ of the donor forms the disk; for equal mass mergers, $\lesssim 10 \%$ of donor and accretor 


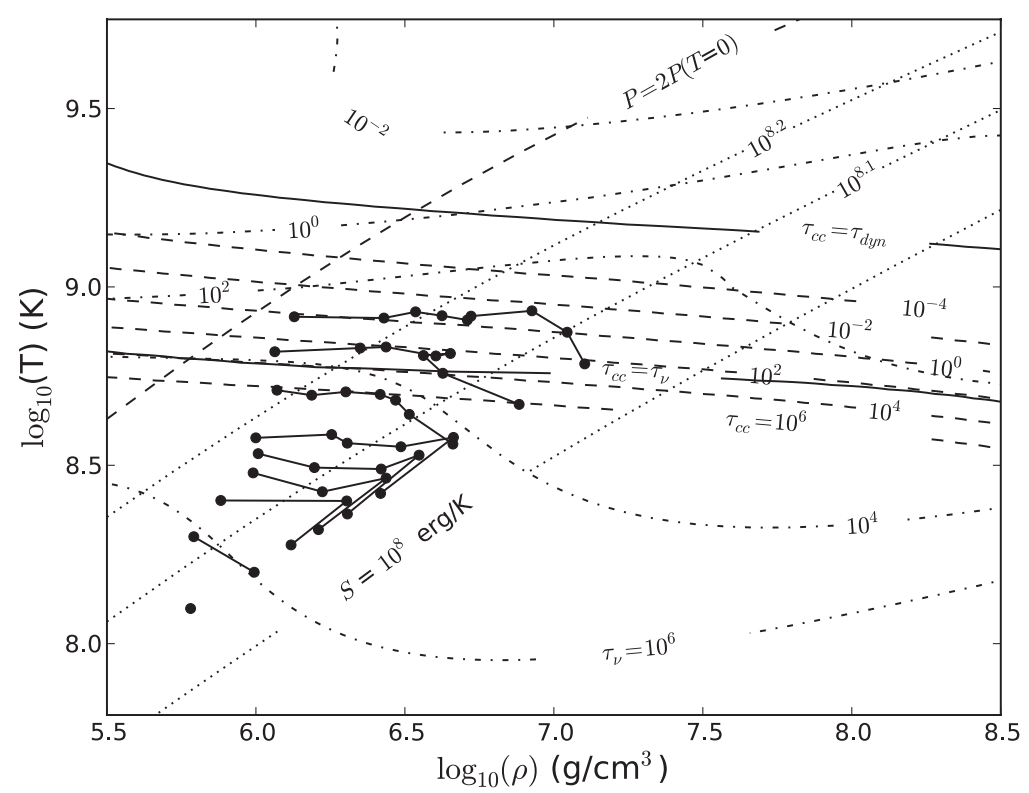

Figure 1. Merger remnant maximum temperature and corresponding density. Black points are merger remnants, and lines connect points with the same accretor mass (from bottom to top, $0.4,0.5,0.55,0.6,0.65,0.7,0.8,0.9$ and $1.0 \mathrm{M}_{\odot}$ ). Maximum temperatures of nearly equal mass mergers have been adjusted to account for mixing in their convectively unstable cores. Also shown are contours of neutrino cooling timescale $\tau_{\nu}$, carbon fusion timescale $\tau_{\text {cc }}$ and entropy $S$. The $\tau_{\nu}=\tau_{\mathrm{cc}}$ and $\tau_{\mathrm{dyn}}=\tau_{\mathrm{cc}}$ lines denote where the carbon fusion timescale balances the neutrino cooling and dynamical timescales, respectively. The $P=2 P(T=0)$ line marks the upper bound to where matter is degenerate.

go into the disk. Other mergers fall on a continuum between these two extremes. Some results important to the possibility of the remnant exploding are summarized below:

- The maximum temperature $T_{\max }$ of the system is nearly independent of mass ratio, and is within $20 \%$ of $a G M_{\mathrm{a}} m_{\mathrm{H}} /\left(R_{\mathrm{a}} k_{B}\right)$ (i.e. it scales with the gravitational potential of the accreting WD before merger), with $a=0.270$. Most of the equal and nearly equal mass mergers have convectively unstable cores. Artificially mixing these cores to make them isentropic decreases their maximum temperatures by $10-40 \%$ (see Figure 1).

- The density at maximum temperature $\rho\left(T_{\max }\right)$ of the system is within $52 \%$ of central density of the donor, $\rho_{\mathrm{d}, \mathrm{c}}$.

- The central density of the remnant $\rho_{\mathrm{c}}$ is within $67 \%$ of $\rho_{\mathrm{a}, \mathrm{c}}$, the central density of the accretor. The central temperature $T_{\mathrm{c}}$ for unequal mass mergers is significantly lower than $T_{\max }$. Since our conditions are far from the pyconuclear regime, temperature is more important than density, and so $T_{\max }$ is more relevant to post merger evolution than $T_{\mathrm{c}}$, despite $\rho_{\mathrm{c}}$ being higher than $\rho\left(T_{\max }\right)$.

- The enclosed mass inside the radius of highest temperature $M_{\mathrm{enc}}\left(T_{\max }\right)$ is within $35 \%$ of $M_{\mathrm{a}}$, the mass of the accretor, when $\rho_{\mathrm{d}, \mathrm{c}} / \rho_{\mathrm{a}, \mathrm{c}}<0.6$, i.e. for unequal mass mergers the hottest point is near the core-envelope interface. $M_{\text {enc }}\left(T_{\max }\right)$ is zero when $\rho_{\mathrm{d}, \mathrm{c}} / \rho_{\mathrm{a}, \mathrm{c}}>0.6$, i.e. the hottest point is at the center for nearly equal mass mergers. The cut-off at $\rho_{\mathrm{d}, \mathrm{c}} / \rho_{\mathrm{a}, \mathrm{c}}=0.6$ is sharp, suggesting that this ratio defines whether or not the merger is "equal mass". 


\section{Implications for the Sub-M $\mathbf{M}_{\mathrm{Ch}}$ Merger SNe Ia Channel}

For Figure 1 we have replicated Van Kerkwijk et al.'s Figure 1, and added our simulated merger remnants. Although several remnants are above the $\tau_{\mathrm{cc}}=\tau_{\nu}$ runaway line, none reach a carbon burning timescale shorter than the accretion timescale of several hours. Accretion of the disk onto the core is therefore of most immediate consequence. Accretion results in adiabatic compressional heating, and may increase the density by up to an order of magnitude. Transposing the points on Figure 1 one order of magnitude in density along a contour of constant entropy results in all remnants with accretor masses $\gtrsim 0.55 \mathrm{M}_{\odot}$ reaching ignition $\left(\tau_{\mathrm{cc}}=\tau_{\nu}\right)$. Those remnants with small mass ratios $q \lesssim 0.5$ will likely not explode, since as the runaway progresses, they would lose degeneracy around the time the fusion timescale becomes equal to the degeneracy timescale $\left(\tau_{\text {dyn }}=\tau_{\text {cc }}\right)$. For all others, explosion seems, at least in principle, possible.

A more accurate depiction of post merger evolution would require taking into consideration the the full viscous evolution, angular momentum transport, heating and compression throughout the remnant (see Shen et al. 2011 for initial studies). Likewise, the later, rapid nuclear runaway requires simulation to see if it can lead to detonation (Chang, White \& Van Kerkwijk, in prep.). As noted earlier, merger remnants with their hottest point at the center have $\rho_{\mathrm{d}, \mathrm{c}} / \rho_{\mathrm{a}, \mathrm{c}}>0.6$ (corresponding to approximately $\left.M_{\mathrm{a}}-M_{\mathrm{d}} \lesssim 0.08 \mathrm{M}_{\odot}\right)$. If this is required for an explosion, only a fraction of our remnants will explode. It is still possible, however, that some systems will experience off-center detonations as a result of compressional heating. The heaviest of our equal-mass mergers may also explode as they merge, as described in Pakmor et al. (2010, 2011).

Nevertheless, Figure 1 suggests that the sub- $\mathrm{M}_{\mathrm{Ch}}$ merger channel for SNe Ia merits further investigation.

\section{References}

Balsara, D. S. 1995, J. Comput. Phys., 121, 357

Di Stefano, R. 2010, ApJ, 712, 728

Di Stefano, R. 2010, ApJ, 719, 474

Fuller, J. \& Lai, D. 2011, arxiv:1108.4910

Gilfanov, M. \& Bogdán, Á. 2010, Nature, 463, 924

Guerrero, J., García-Berro, E., \& Isern, J. 2004, A\&A, 413, 257

Guillochon, J., Dan, M., Ramirez-Ruiz, E., \& Rosswog, S. 2010, ApJ (Letters), 709, L64

Howell, D. A. 2011, Nat. Commun., 2, 350

Lorén-Aguilar, P., Isern, J., \& García-Berro, E. 2009, A\&A, 500, 1193

Maoz, D. 2008, MNRAS, 384, 267

Marsh, T. R., Nelemans, G., \& Steeghs, D. 2004, MNRAS, 350, 113

Monaghan, J. J. 1992, ARAA, 30, 543

Pakmor, R., Hachinger, S., Röpke, F. K., \& Hillebrandt, W. 2011, A\& A, 528, A117

Pakmor, R., Kromer, M., Röpke, F. K., Sim, S. A., Ruiter, A. J., \& Hillebrandt, W. 2010, Nature, 463, 61

Ruiter, A. J., Belczynski, K., \& Fryer, C. 2009, ApJ, 699, 2026

Shen, K. J., Bildsten, L., Kasen, D., \& Quataert, E. 2011, arxiv:1108.4036

Sim, S. A., Röpke, F. K., Hillebrandt, W., Kromer, M., Pakmor, R., Fink, M., Ruiter, A. J., \& Seitenzahl, I. R. 2010, ApJ (Letters), 714, L52

Timmes, F. 2010, Helmholtz Stellar Equation of State, available at http://cococubed.asu.edu/

Timmes, F. X. \& Swesty, F. D. 2000, ApJS, 126, 501

Van Kerkwijk, M. H., Chang, P., \& Justham, S. 2010, ApJ(Letters), 722, L157

Wadsley, J. W., Stadel, J., \& Quinn, T. 2004, New Astron., 9, 137

Woosley, S. E. \& Kasen, D. 2011, ApJ, 734, 38 